\title{
SOME CONVERGENCE ACCELERATION PROCESSES FOR A CLASS OF VECTOR SEQUENCES
}

Abstract. Let $\left(S_{n}\right)$ be some vector sequence, converging to $S$, satisfying

$$
S_{n}-S \sim \varrho^{n} n^{\theta}\left(\beta_{0}+\beta_{1} n^{-1}+\beta_{2} n^{-2}+\ldots\right), \quad 0<|\varrho|<1, \theta<0,
$$

where $\beta_{0}(\neq 0), \beta_{1}, \ldots$ are constant vectors independent of $n$. The purpose of this paper is to provide acceleration methods for these vector sequences. Comparisons are made with some known algorithms. Numerical examples are also given.

1. Introduction. We shall denote by $\mathcal{L}$ the following set of vector sequences $\left(S_{n}\right)$ converging to $S$ :

$$
\begin{aligned}
\mathcal{L}=\left\{\left(S_{n}\right): S_{n}-S \sim \varrho^{n} n^{\theta}\left(\beta_{0}+\beta_{1} n^{-1}+\beta_{2} n^{-2}+\right.\right. & \ldots), \\
& 0<|\varrho|<1, \theta<0,
\end{aligned}
$$

where $\beta_{0}(\neq 0), \beta_{1}, \ldots$ are constant vectors independent of $\left.n\right\}$.

We propose an original algorithm. An extension of Aitken's $\Delta^{2}$-process [1] as well as iterations of this algorithm are studied. Convergence theorems for the sequences of $\mathcal{L}$ are proved. Some insight in numerical properties of the methods is given. The first part is devoted to the scalar case.

2. The A-algorithm (the scalar case). In this section, we shall consider the case of scalar sequences.

Given any sequence $\left(S_{n}\right)$, we set

$$
\begin{aligned}
u_{0, n} & =\frac{\Delta S_{n}}{\Delta S_{n+1}}, \quad A_{0, n}=S_{n}, \quad n=0,1,2, \ldots, \\
u_{k+1, n} & =G\left(u_{k, n}, u_{k, n}\right), \quad k=0,1,2, \ldots, \\
A_{k+1, n} & =G\left(A_{k, n}, u_{k, n}\right), \quad k=0,1,2, \ldots,
\end{aligned}
$$

1991 Mathematics Subject Classification: 65B05, 65H10.

Key words and phrases: convergence acceleration, vector extrapolation methods. 
where the transform $G$ transforms a sequence $\left(S_{n}\right)$ and an auxiliary sequence $\left(x_{n}\right)$ into $G\left(S_{n}, x_{n}\right)$ such that

$$
G\left(S_{n}, x_{n}\right)=S_{n+1}-\frac{\Delta S_{n}}{1-x_{n}} .
$$

As a matter of convenience, we shall write

$$
G_{n}=G\left(S_{n}, x_{n}\right) .
$$

Let $L$ be the set of scalar sequences $\left(S_{n}\right)$ satisfying

$$
S_{n}-S \sim \varrho^{n} n^{\theta}\left(\beta_{0}+\beta_{1} n^{-1}+\beta_{2} n^{-2}+\ldots\right), \quad 0<|\varrho|<1, \theta<0,
$$

where $\beta_{0}(\neq 0), \beta_{1}, \beta_{2}, \ldots$ are independent of $n$.

Let us recall that for two sequences $\left(S_{n}\right)$ and $\left(T_{n}\right)$ which converge to the same limit $S$, the sequence $\left(T_{n}\right)$ is said to converge faster than $\left(S_{n}\right)$ if

$$
\lim _{n \rightarrow \infty}\left(T_{n}-S\right) /\left(S_{n}-S\right)=0 .
$$

TheOREM 2.1. The A-algorithm accelerates the convergence of linearly converging sequences. That is, for any linearly converging sequence $\left(S_{n}\right)$ and for any fixed $k \in \mathbb{N},\left(A_{k+1, n}\right)$ converges faster than $\left(A_{k, n}\right)$.

Remarks. Let us recall that a sequence $\left(S_{n}\right)$ converges linearly [11, p. 6] to $S$ if

- there exists $N \in \mathbb{N}$ such that $S_{n} \neq S$ for all $n \geq N$,

- there exists a number $r$ such that $0<|r|<1$ and

$$
\lim _{n \rightarrow \infty}\left(S_{n+1}-S\right) /\left(S_{n}-S\right)=r .
$$

The proof of Theorem 2.1 follows from a well known result (see Theorem 1.8 of [4]). Let us recall that $A_{1, n}$ is Aitken's $\Delta^{2}$-process.

Lemma 2.1. For any sequence $\left(S_{n}\right) \in L$ and for a choice of $\left(x_{n}\right)$ such that $x_{n} \sim 1 / \varrho+d_{1} n^{-1}+d_{2} n^{-2}+\ldots$, where $d_{1}, d_{2}, \ldots$ are independent of $n$, $\left(G_{n}\right)$ converges faster than $\left(S_{n}\right)$. Moreover, $\left(G_{n}\right) \in L$.

Proof. We get

$$
G_{n}-S=S_{n+1}-S-\frac{\Delta S_{n}}{1-x_{n}}
$$

and

$$
\frac{G_{n}-S}{S_{n}-S}=\frac{e_{n+1}}{e_{n}}-\frac{1}{1-x_{n}}\left(\frac{e_{n+1}}{e_{n}}-1\right), \quad \text { where } \quad e_{n}=S_{n}-S .
$$

Hence

since

$$
\lim _{n \rightarrow \infty}\left(G_{n}-S\right) /\left(S_{n}-S\right)=0 \text { if } \lim _{n \rightarrow \infty} x_{n}=1 / \varrho,
$$

$$
\lim _{n \rightarrow \infty} e_{n+1} / e_{n}=\varrho .
$$


There exist constants $\gamma_{0}, \gamma_{1}, \ldots$, such that

$$
\Delta S_{n} \sim \varrho^{n} n^{\theta}\left(\gamma_{0}+\gamma_{1} n^{-1}+\ldots\right) .
$$

By the asymptotic expansion of $x_{n}$, we have

$$
\frac{1}{1-x_{n}} \sim \frac{\varrho}{\varrho-1}\left(1+c_{1} n^{-1}+c_{2} n^{-2}+\ldots\right)
$$

where $c_{1}, c_{2}, \ldots$ are constants, e.g. $c_{1}=\varrho d_{1} /(\varrho-1), c_{2}=c_{1}^{2}+\varrho d_{2} /(\varrho-1)$.

Thus

$$
\frac{\Delta S_{n}}{1-x_{n}} \sim \frac{\varrho^{n+1} n^{\theta}}{\varrho-1}\left(\gamma_{0}+\gamma_{1} n^{-1}+\ldots\right)\left(1+c_{1} n^{-1}+\ldots\right),
$$

and $\frac{\Delta S_{n}}{1-x_{n}} \sim \varrho^{n} n^{\theta}\left(\gamma_{0}^{\prime}+\gamma_{1}^{\prime} n^{-1}+\ldots\right)$, where $\gamma_{0}^{\prime}, \gamma_{1}^{\prime}, \ldots$ are independent of $n$.

There also exist constants $\lambda_{0}, \lambda_{1}, \ldots$, such that

$$
S_{n+1}-S \sim \varrho^{n} n^{\theta}\left(\lambda_{0}+\lambda_{1} n^{-1}+\ldots\right) .
$$

Hence $G_{n}-S \sim \varrho^{n+1} n^{\theta-1}\left(\lambda_{0}^{\prime}+\lambda_{1}^{\prime} n^{-1}+\ldots\right)$, where $\lambda_{0}^{\prime}, \lambda_{1}^{\prime}, \ldots$ are constants. Therefore $\left(G_{n}\right) \in L$.

THEOREM 2.2. For any sequence $\left(S_{n}\right) \in L$ and for any fixed $k \in \mathbb{N}$, $\left(A_{k+1, n}\right)$ converges faster than $\left(A_{k, n}\right)$.

Pr o of. Since

$$
u_{k+1, n}=G\left(u_{k, n}, u_{k, n}\right),
$$

it can easily be proved by induction that $u_{k, n} \sim 1 / \varrho+d_{1} n^{-1}+d_{2} n^{-2}+\ldots$, where $d_{1}, d_{2}, \ldots$ are independent of $n$. By Lemma 2.1, $\left(A_{k+1, n}\right)$ converges faster than $\left(A_{k, n}\right)$.

3. The A-algorithm (the vector case). Let $x=\left(x_{1}, \ldots, x_{d}\right)^{T}$, $y=\left(y_{1}, \ldots, y_{d}\right)^{T}$ be $d$-dimensional real vectors. We define

$$
\|x\|=\sqrt{\sum x_{i}^{2}} \text { and }(x, y)=\sum x_{i} y_{i} .
$$

Using (1), we extend the $A$-algorithm to vector sequences $\left(S_{n}\right)$ as follows:

$$
\begin{aligned}
u_{0, n} & =\frac{\left(\Delta S_{n}, \Delta S_{n+1}\right)}{\left\|\Delta S_{n+1}\right\|^{2}}, & A_{0, n}=S_{n}, \quad n=0,1,2, \ldots, \\
u_{k+1, n} & =G\left(u_{k, n}, u_{k, n}\right), & k=0,1,2, \ldots, \\
A_{k+1, n} & =G^{T}\left(A_{k, n}, u_{k, n}\right), & k=0,1,2, \ldots,
\end{aligned}
$$

where the transform $G^{T}$ transforms a vector sequence $\left(S_{n}\right)$ and an auxiliary sequence $\left(x_{n}\right)$ into $G^{T}\left(S_{n}, x_{n}\right)$ such that

$$
G^{T}\left(S_{n}, x_{n}\right)=S_{n+1}-\frac{\Delta S_{n}}{1-x_{n}} .
$$


As a matter of convenience, we shall write

$$
G_{n}^{T}=G^{T}\left(S_{n}, x_{n}\right) .
$$

This algorithm is named the vector $A$-algorithm (VAA for short).

Given two vector sequences $\left(S_{n}\right)$ and $\left(T_{n}\right)$ which converge to the same limit $S$, the sequence $\left(T_{n}\right)$ is said to converge faster than $\left(S_{n}\right)$ if

$$
\lim _{n \rightarrow \infty}\left\|T_{n}-S\right\| /\left\|S_{n}-S\right\|=0 .
$$

Lemma 3.1. For any sequence $\left(S_{n}\right) \in \mathcal{L}$, if $x_{n} \sim 1 / \varrho+d_{1} n^{-1}+d_{2} n^{-2}+$ $\ldots$, where $d_{1}, d_{2}, \ldots$ are independent of $n$, then $\left(G_{n}^{T}\right)$ converges faster than $\left(S_{n}\right)$. Moreover, $\left(G_{n}^{T}\right) \in \mathcal{L}$.

Proof. We get

$$
G_{n}^{T}-S=S_{n+1}-S-\frac{\Delta S_{n}}{1-x_{n}} .
$$

There exist constant vectors $\beta_{1}^{\prime}, \beta_{2}^{\prime}, \ldots$ such that

$$
\Delta S_{n} \sim \varrho^{n} n^{\theta}\left[(\varrho-1) \beta_{0}+\beta_{1}^{\prime} n^{-1}+\beta_{2}^{\prime} n^{-2}+\ldots\right] .
$$

By the asymptotic expansion of $x_{n}$, we have

$$
\frac{1}{1-x_{n}} \sim \frac{\varrho}{\varrho-1}\left(1+c_{1} n^{-1}+c_{2} n^{-2}+\ldots\right),
$$

where $c_{1}, c_{2}, \ldots$ are constants, e.g. $c_{1}=\varrho d_{1} /(\varrho-1), c_{2}=c_{1}^{2}+\varrho d_{2} /(\varrho-1)$.

Thus

$$
\frac{\Delta S_{n}}{1-x_{n}} \sim \frac{\varrho^{n+1} n^{\theta}}{\varrho-1}\left[(\varrho-1) \beta_{0}+\beta_{1}^{\prime} n^{-1}+\ldots\right]\left(1+c_{1} n^{-1}+\ldots\right),
$$

and

$$
\frac{\Delta S_{n}}{1-x_{n}} \sim \varrho^{n+1} n^{\theta}\left(\beta_{0}+\gamma_{1} n^{-1}+\gamma_{2} n^{-2}+\ldots\right),
$$

where $\gamma_{1}, \gamma_{2}, \ldots$ are constant vectors.

There also exist constant vectors $\lambda_{1}, \lambda_{2}, \ldots$ such that

$$
S_{n+1}-S \sim \varrho^{n+1} n^{\theta}\left(\beta_{0}+\lambda_{1} n^{-1}+\lambda_{2} n^{-2}+\ldots\right) .
$$

Hence $G_{n}^{T}-S \sim \varrho^{n+1} n^{\theta-1}\left(\lambda_{0}^{\prime}+\lambda_{1}^{\prime} n^{-1}+\ldots\right)$, where $\lambda_{0}^{\prime}, \lambda_{1}^{\prime}, \ldots$ are constant vectors.

Therefore $\left(G_{n}^{T}\right) \in \mathcal{L}$ and $\lim _{n \rightarrow \infty}\left\|G_{n}^{T}-S\right\| /\left\|S_{n}-S\right\|=0$.

Theorem 3.1. For any sequence $\left(S_{n}\right) \in \mathcal{L}$ and for any fixed $k \in \mathbb{N}$, $\left(A_{k+1, n}\right)$ converges faster than $\left(A_{k, n}\right)$.

Proof. There exist constant vectors $\beta_{1}^{\prime}, \beta_{2}^{\prime}, \ldots$ such that

$$
\Delta S_{n} \sim \varrho^{n} n^{\theta}\left[(\varrho-1) \beta_{0}+\beta_{1}^{\prime} n^{-1}+\beta_{2}^{\prime} n^{-2}+\ldots\right] .
$$


There also exist constants $\lambda_{1}, \lambda_{2}, \ldots$ such that

$$
\left(\Delta S_{n}, \Delta S_{n+1}\right) \sim \varrho^{2 n+1} n^{2 \theta}\left[(\varrho-1)^{2}\left(\beta_{0}, \beta_{0}\right)+\lambda_{1} n^{-1}+\lambda_{2} n^{-2}+\ldots\right] .
$$

Hence

$$
u_{0, n} \sim 1 / \varrho+\gamma_{1} n^{-1}+\gamma_{2} n^{-2}+\ldots,
$$

where $\gamma_{1}, \gamma_{2}, \ldots$ are independent of $n$.

Since $u_{k+1, n}=G\left(u_{k, n}, u_{k, n}\right)$, it can easily be proved by induction that $u_{k, n} \sim 1 / \varrho+d_{1} n^{-1}+d_{2} n^{-2}+\ldots$, where $d_{1}, d_{2}, \ldots$ are independent of $n$. By Lemma 3.1, $\left(A_{k+1, n}\right)$ converges faster than $\left(A_{k, n}\right)$.

4. Some extensions of Aitken's $\Delta^{2}$-process for vector sequences. Some authors extended Aitken's $\Delta^{2}$-process to vector sequences:

Irons and Tuck [6]:

$$
T_{n}:=S_{n}-\frac{\left(\Delta S_{n}, \Delta^{2} S_{n}\right)}{\left(\Delta^{2} S_{n}, \Delta^{2} S_{n}\right)} \Delta S_{n} ;
$$

Graves-Morris [5]:

$$
T_{n}:=S_{n+1}-\frac{\left(\Delta S_{n}, \Delta S_{n}\right)}{\left(\Delta S_{n}, \Delta^{2} S_{n}\right)} \Delta S_{n+1} .
$$

We define a vector Aitken's $\Delta^{2}$-process ( $V A \Delta^{2}$ for short) as the first step of the vector $A$-algorithm defined by (2).

This algorithm can be written as follows:

$$
T_{n}:=S_{n+1}-\frac{\left(\Delta S_{n+1}, \Delta S_{n+1}\right)}{\left(\Delta S_{n+1}, \Delta^{2} S_{n}\right)} \Delta S_{n} .
$$

Many authors: Wynn [13], Brezinski [3], Weniger [10], Bhowmick, Bhattacharya and Roy [2] showed that the repeated application of an extrapolation algorithm can lead to improvements either of the results or of the stability.

So we define iterations of the vector Aitken's $\Delta^{2}$-process. The algorithm obtained $\left(I V A \Delta^{2}\right.$ for short) can be written as follows for a sequence $\left(S_{n}\right)$ :

$$
\begin{aligned}
B_{0, n} & =S_{n}, \quad n=0,1,2, \ldots, \\
v_{k, n} & =\frac{\left(B_{k, n+1}-B_{k, n}, B_{k, n+2}-B_{k, n+1}\right)}{\left\|B_{k, n+2}-B_{k, n+1}\right\|^{2}}, \quad k=0,1,2, \ldots, \\
B_{k+1, n} & =G^{T}\left(B_{k, n}, v_{k, n}\right), \quad k=0,1,2, \ldots
\end{aligned}
$$

THEOREM 4.1. Let $\left(S_{n}\right)$ be a sequence of $\mathcal{L}$ and assume that $\left(B_{k, n}\right)$ is the sequence generated by the $k$ th iteration of the vector $\Delta^{2}$-process applied to $\left(S_{n}\right)$. Then $\left(B_{k+1, n}\right)$ converges faster than $\left(B_{k, n}\right)$. 
Proof. It can easily be proved by induction that

$$
v_{k, n} \sim 1 / \varrho+d_{1} n^{-1}+d_{2} n^{-2}+\ldots,
$$

where $d_{1}, d_{2}, \ldots$ are independent of $n$. By Lemma $3.1,\left(B_{k+1, n}\right)$ converges faster than $\left(B_{k, n}\right)$.

5. Comparisons and numerical examples. For a $d$-dimensional real vector $x=\left(x_{1}, \ldots, x_{d}\right)^{T}$, we define

$$
\|x\|_{\infty}=\max \left\{\left|x_{1}\right|, \ldots,\left|x_{d}\right|\right\} .
$$

Osada [7] extended Levin's transforms to vector sequences.

We shall compare these algorithms and the vector $\varepsilon$-algorithm (VEA) [12] to ours. For a vector sequence $\left(x_{n}\right)$ and for a vector sequence transformation $\left(x_{n}\right) \rightarrow\left(y_{n}\right)$, the number of significant digits of $y_{n}$ is defined by

$$
-\log _{10}\left\|y_{n}-x\right\|_{\infty},
$$

when $x$ is the limit of $\left(x_{n}\right)$. We shall apply the algorithms to two systems of nonlinear equations whose Jacobian matrix is singular at zero.

The results given by vector Levin's transforms and the vector $\varepsilon$-algorithm are similar (see [7]). So this class of algorithms will be represented by the vector $\varepsilon$-algorithm.

The numerical results are obtained in double precision with 16 significant digits.

EXAmple 5.1. Consider the system of nonlinear equations [8]

$$
\left\{\begin{aligned}
x^{2}-x y+y^{2}+x-2 & =0, \\
3 x^{2}+2 x y+2 y-7 & =0 .
\end{aligned}\right.
$$

The only real solution is $(1,1)^{T}$ and the Jacobian at this point is zero. Newton's iteration is explicitly given as

$x_{n+1}=\frac{1}{\Delta}\left(5 x_{n}^{3}+2 x_{n}^{2}+11 x_{n}-6 x_{n}^{2} y_{n}-2 x_{n} y_{n}^{2}-2 x_{n} y_{n}+2 y_{n}^{2}-14 y_{n}+4\right)$,

$y_{n+1}=\frac{1}{\Delta}\left(3 x_{n}^{2}+2 x_{n}+5 x_{n}^{2} y_{n}-6 x_{n} y_{n}^{2}+2 x_{n} y_{n}-2 y_{n}^{3}-11 y_{n}+7\right)$,

with

$$
\Delta=10 x_{n}^{2}+6 x_{n}-12 x_{n} y_{n}-4 y_{n}^{2}-2 y_{n}+2 .
$$

If we set $S_{n}=\left(x_{n}, y_{n}\right)^{T}$, then $\left(S_{n}\right)$ converges linearly to $(0,0)^{T}$ with ratio $1 / 2$ (see [8]). We give below the numbers of significant digits by applying the algorithms with $S_{0}=(0,0)^{T}$. 


\begin{tabular}{cccrr}
\hline$n$ & Base & $I V A \Delta^{2}$ & $V A A$ & $V E A$ \\
\hline 3 & 0.04 & 0.04 & 0.04 & 0.04 \\
4 & 0.49 & 0.49 & 0.49 & 0.49 \\
5 & 0.82 & 0.82 & 0.82 & 0.82 \\
6 & 1.14 & 1.21 & 1.21 & 1.21 \\
7 & 1.44 & 3.62 & 3.50 & 3.10 \\
8 & 1.74 & 5.55 & 5.30 & 5.05 \\
9 & 2.04 & 7.82 & 8.23 & 8.10 \\
10 & 2.35 & 10.40 & 10.77 & 10.36 \\
11 & 2.64 & 12.18 & 12.68 & 11.37 \\
12 & 2.96 & 13.82 & 13.80 & 12.92 \\
\hline & & & &
\end{tabular}

EXAMPLE 5.2. Consider the system of nonlinear equations [9]

$$
\left\{\begin{aligned}
x+x y+y^{2} & =0 \\
x^{2}-2 x+y^{2} & =0 \\
x+z^{2} & =0 .
\end{aligned}\right.
$$

The only real solution is $(0,0,0)^{T}$ and the Jacobian at this point is zero. Newton's iteration is explicitly given as

$$
\begin{aligned}
x_{n+1} & =\frac{-x_{n}^{3}-2 x_{n}^{2} y_{n}+x_{n} y_{n}^{2}}{2 x_{n}+6 y_{n}-2 x_{n}^{2}-4 x_{n} y_{n}+2 y_{n}^{2}}, \\
y_{n+1} & =\frac{y_{n}}{2}+\frac{x_{n} y_{n}+x_{n}^{2}}{2 x_{n}+6 y_{n}-2 x_{n}^{2}-4 x_{n} y_{n}+2 y_{n}^{2}}, \\
z_{n=1} & =\frac{z_{n}}{2}-\frac{x_{n+1}}{2 z_{n}} .
\end{aligned}
$$

If we set $S_{n}=\left(x_{n}, y_{n}, z_{n}\right)^{T}$, then $\left(S_{n}\right)$ converges linearly to $(0,0,0)^{T}$ with ratio $1 / 2$ (see [9]). We give below the numbers of significant digits by applying the algorithms with $S_{0}=(0.1,0.5,1.0)^{T}$.

\begin{tabular}{cccrr}
\hline$n$ & Base & $I V A \Delta^{2}$ & $V A A$ & $V E A$ \\
\hline 1 & 0.30 & 0.30 & 0.30 & 0.30 \\
2 & 0.60 & 0.60 & 0.60 & 0.60 \\
3 & 0.91 & 2.47 & 2.47 & 2.47 \\
4 & 1.21 & 5.01 & 4.70 & 4.99 \\
5 & 1.51 & 5.67 & 5.53 & 5.49 \\
6 & 1.81 & 6.55 & 6.30 & 6.29 \\
7 & 2.11 & 10.20 & 9.80 & 9.69 \\
8 & 2.41 & 11.50 & 10.30 & 11.02 \\
\hline
\end{tabular}

6. Conclusions. The vector algorithms proposed in this paper give similar results to some known algorithms. Moreover, each column of the arrays converges faster than the previous one. That is, given any algorithm $\left(T_{k, n}\right)$ introduced here, for any $k,\left(T_{k+1, n}\right)$ converges faster than $\left(T_{k, n}\right)$. 
The error analysis has not been studied in this paper. It will be the matter of further research.

\section{References}

[1] A. C. Aitken, On Bernoulli's numerical solution of algebraic equations, Proc. Roy. Soc. Edinburgh 46 (1926), 289-305.

[2] S. Bhowmick, R. Bhattacharya and D. Roy, Iterations of convergence accelerating nonlinear transforms, Comput. Phys. Comm. 54 (1989), 31-46.

[3] C. Brezinski, Algorithmes d'Accélération de la Convergence, Etude Numérique, Editions Technip, Paris, 1978.

[4] C. Brezinski and M. Redivo Zaglia, Extrapolation Methods. Theory and Practice, Math. Stud. in Comput. Math. 2, North-Holland, 1991.

[5] P. R. Graves-Morris, Extrapolation method for vector sequences, Numer. Math. 61 (1992), 475-487.

[6] B. M. Irons and R. C. Tuck, A version of the Aitken accelerator for computer iteration, Internat. J. Numer. Methods Engrg. 1 (1969), 275-277.

[7] N. Osada, Extensions of Levin's transformations to vector sequences, Numer. Algorithms 2 (1992), 121-132.

[8] L. B. Rall, Convergence of the Newton process to multiple solutions, Numer. Math. 9 (1966), 23-37.

[9] G. W. Reddien, Newton's method and high order singularities, Comput. Math. Appl. 5 (1979), 79-86.

[10] E. J. Weniger, On the derivation of iterated sequence transformations for the acceleration of convergence and the summation of divergent series, Comput. Phys. Comm. 64 (1991), 19-45.

[11] J. Wimp, Sequence Transformations and their Applications, Academic Press, New York, 1981.

[12] P. Wynn, Acceleration techniques for iterated vector and matrix problems, Math. Comp. 16 (1962), 301-322.

[13] —, Transformations de séries à l'aide de l'e-algorithme, C. R. Acad. Sci. Paris Sér. A 275 (1972), 1351-1353.

Laboratoire d'Analyse Numérique et d'Optimisation

Université des Sciences et Technologies de Lille

59655 Villeneuve d'Ascq Cedex, France 